\title{
Clinical Characteristics, Management, and Potential Biomarkers of Endocrine Dysfunction Induced by Immune Checkpoint Inhibitors
}

\author{
Shintaro Iwama ${ }^{1}$, Tomoko Kobayashi ${ }^{1}$, Hiroshi Arima ${ }^{2}$ \\ ${ }^{1}$ Department of Endocrinology and Diabetes, Nagoya University Hospital; ${ }^{2}$ Department of Endocrinology and Diabetes, Nagoya \\ University Graduate School of Medicine, Nagoya, Japan
}

Immune-related adverse events (irAEs) affecting the endocrine glands are among the most frequent irAEs induced by immune checkpoint inhibitors (ICIs) and include hypopituitarism, primary adrenal insufficiency, thyrotoxicosis, hypothyroidism, hypoparathyroidism, and type 1 diabetes mellitus. Since the incidence and clinical features of endocrine irAEs vary according to the ICI used, it is important to understand the characteristics of these irAEs and to manage each one appropriately. Since some endocrine irAEs, including adrenal crisis and diabetic ketoacidosis, are potentially life-threatening, predicting the risk of endocrine irAEs before their onset is critical. Several autoantibodies have been detected in patients who develop endocrine irAEs, among which anti-thyroid antibodies may be predictive biomarkers of thyroid dysfunction. In this review, we describe the clinical features of each endocrine irAE induced by ICIs and discuss their potential biomarkers, including autoantibodies.

Keywords: Hypopituitarism; Hypophysitis; Adrenal insufficiency; Thyrotoxicosis; Hypothyroidism; Hypoparathyroidism; Diabetes mellitus, type 1; Immune checkpoint inhibitors; Autoantibodies

\section{INTRODUCTION}

Immune checkpoint inhibitors (ICIs) are widely used to treat several types of advanced malignancies, including malignant melanoma and non-small cell lung carcinoma. However, ICIs can cause characteristic adverse events, termed immune-related adverse events (irAEs), in a variety of organs including the lung,

Received: 22 February 2021, Revised: 10 March 2021,

Accepted: 24 March 2021

Corresponding authors: Shintaro Iwama

Department of Endocrinology and Diabetes, Nagoya University Hospital,

65 Tsurumai-cho, Showa-ku, Nagoya 466-8550, Japan

Tel: +81-52-744-2142, Fax: $+81-52-744-2212$

E-mail: siwama@med.nagoya-u.ac.jp

Hiroshi Arima

Department of Endocrinology and Diabetes, Nagoya University Graduate School of Medicine, 65 Tsurumai-cho, Showa-ku, Nagoya, 466-8550, Japan

Tel: +81-52-744-2194, Fax: +81-52-744-2212

E-mail: arima105@med.nagoya-u.ac.jp skin, liver, colon, and endocrine glands [1,2]. Endocrine irAEs include dysfunction of the pituitary, adrenal gland, thyroid, parathyroid, and pancreatic $\beta$ cells [3-6] and can cause lifethreatening consequences, such as adrenal crisis and diabetic ketoacidosis. On the other hand, it has been reported that the development of pituitary $[7,8]$ or thyroid [8-10] dysfunction is associated with better outcomes after ICI treatment. Therefore,

\section{Copyright $@ 2021$ Korean Endocrine Society}

This is an Open Access article distributed under the terms of the Creative Commons Attribution Non-Commercial License (https://creativecommons.org/ licenses/by-nc/4.0/) which permits unrestricted non-commercial use, distribution, and reproduction in any medium, provided the original work is properly cited. 
endocrine irAEs should be diagnosed promptly and managed appropriately. Establishing biomarkers is also important for identifying patients at risk of developing endocrine irAEs before initiating ICI treatment.

\section{PITUITARY DYSFUNCTION}

\section{Epidemiology}

Pituitary dysfunction is induced by all ICI types, including anticytotoxic T-lymphocyte antigen 4 (CTLA-4), anti-programmed cell death-1 (PD-1), and anti-programmed cell death-1 ligand 1 (PD-L1) antibodies. A systematic review reported that the incidences of pituitary dysfunction induced by anti-CTLA-4, antiPD-1, combination anti-CTLA-4/anti-PD-1, and anti-PD-L1 antibodies were $3.2 \%, 0.4 \%, 6.4 \%$, and $<0.1 \%$, respectively [11]. In contrast, our prospective study indicated much higher incidences of pituitary dysfunction induced by anti-CTLA-4 (24\%; 6/25 patients) and anti-PD-1 (6\%; 10/167 patients) antibodies [8], suggesting that pituitary dysfunction may be overlooked by attending physicians due to non-specific symptoms such as general fatigue and appetite loss.

\section{Clinical types}

Pituitary dysfunction induced by ICIs can be classified into two clinical types: isolated adrenocorticotropic hormone (ACTH) deficiency (IAD), which is not associated with pituitary enlargement, and hypophysitis, which is associated with deficiencies in multiple anterior pituitary hormones accompanied by pituitary enlargement [8]. Both types of pituitary dysfunction are always accompanied by ACTH deficiency $[8,12]$. Treatment with an anti-CTLA-4 antibody causes both IAD and hypophysitis, whereas treatment with an anti-PD-1 or PD-L1 antibody causes only IAD [8]. ICI treatment rarely causes central diabetes insipidus due to impaired hypothalamo-neurohypophysial function [12], although a few cases of central diabetes insipidus have been reported $[13,14]$. Most patients treated with an anti-CTLA-4 antibody (ipilimumab) developed pituitary dysfunction within several months (median 10 weeks) after treatment initiation [12]. In contrast, there appears to be no period of greater susceptibility to developing pituitary dysfunction induced by anti-PD-1 or anti-PD-L1 antibodies; pituitary dysfunction can develop several months to over a year after treatment initiation [15-17]. In general, the duration from the initial treatment administration to the onset of pituitary dysfunction is longer with anti-PD-1 than anti-CTLA-4 treatment [8].

\section{Symptoms}

Most symptoms seen in patients with pituitary dysfunction induced by ICIs are caused by secondary adrenal insufficiency. Such symptoms include tiredness, weakness, anorexia, weight loss, digestive symptoms, decreased blood pressure, psychiatric disturbance, fever, hypoglycemic symptoms, joint pain, headache, and visual field disturbance [3].

\section{Endocrinological assessments}

Endocrinological tests show decreased levels of hormones secreted from the anterior pituitary and in the targeted organs [3]. For example, patients with ACTH deficiency show low levels of plasma ACTH and serum cortisol.

\section{Imaging}

Magnetic resonance imaging reveals pituitary enlargement in patients who develop hypophysitis induced by anti-CTLA-4 antibodies, while the enlargement usually improves in a few months $[8,18]$. Patients who develop IAD show no abnormalities in the pituitary gland.

\section{Diagnosis}

Hypopituitarism induced by ICIs is diagnosed when the levels of hormones secreted from the anterior pituitary and in the targeted organs at baseline, or the responses of pituitary hormones in loading tests, are decreased [3].

\section{Treatments and outcomes}

ACTH deficiency should be managed by replacement therapy with physiological doses of hydrocortisone (10 to $20 \mathrm{mg} /$ day) [3]. There is no evidence suggesting any effect of high glucocorticoid doses on treatment outcomes or recovered pituitary dysfunction and enlargement $[7,18]$. Thyroid-stimulating hormone (TSH) deficiency can be managed by replacement therapy with levothyroxine. The dose of levothyroxine should be adjusted according to the serum level of free thyroxine (FT4). When patients simultaneously develop ACTH and TSH deficiencies, hydrocortisone must be administered first, followed by levothyroxine replacement at a low dose ( 12.5 to $25 \mu \mathrm{g} /$ day) 5 to 7 days later. Once the general conditions stabilize after appropriate treatments, the use of ICIs can be considered for patients with pituitary dysfunction. Some studies have shown an association between the development of pituitary dysfunction and ICI treatment outcomes $[7,8]$. 


\section{Pathology and mechanisms}

An autopsy case report described the pathological features of the pituitary gland in a patient who developed hypophysitis induced by tremelimumab (anti-CTLA-4 antibody) [12]. That study demonstrated $\mathrm{T}$ and $\mathrm{B}$ cell infiltration in the anterior pituitary gland, which contained necrotic lesions, and positivity for a complement component of $\mathrm{C} 4 \mathrm{~d}$ in some anterior pituitary cells [12]. In a mouse model of hypophysitis induced by repeated injections of an anti-CTLA-4 antibody, C4d expression was detected in anterior pituitary cells secreting TSH and prolactin, suggesting that injected anti-CTLA-4 (immunoglobulin G1 subclass) can bind to CTLA-4, which was shown to be non-canonically expressed in TSH- and prolactin-secreting cells [19]. Although these data suggest that complement activation may contribute to the development of pituitary inflammation following administration of an anti-CTLA-4 antibody [20], further studies are needed to clarify the mechanism of how pituitary inflammation induced by this ICI leads to autoimmunity against pituitary glands. In addition, the mechanism of IAD induced by ICIs is unknown, since there are no histopathological reports of patients who developed IAD after ICI treatment and no animal models of pituitary dysfunction induced by anti-PD-1 or antiPD-L1 antibodies.

\section{Biomarkers}

One study using serological analysis of recombinant cDNA expression reported that the titers of anti-guanine nucleotide-binding protein $\mathrm{G}$ (olf) subunit alpha and anti-integral membrane protein $2 \mathrm{~B}$ antibodies were increased after the development of pituitary dysfunction [21]. In addition, the titer of the anti-guanine nucleotide-binding protein $\mathrm{G}(\mathrm{olf})$ subunit alpha antibody at baseline was higher in patients with pituitary dysfunction than in those without it [21]. Although these autoantibodies may be a potential biomarker predicting a risk of pituitary dysfunction, the results were validated in only five patients with pituitary dysfunction, including IAD and hypophysitis [21].

Anti-pituitary antibodies (APAs) measured by indirect immunofluorescence in human pituitary sections as a substrate can predict the presence of autoimmunity in pituitary glands [22] and are present in several pituitary diseases [22-25]. APA positivity was observed after the development of hypophysitis induced by ipilimumab in all seven patients evaluated in that study (Table 1) [19]. APAs were also positive at the onset of pituitary dysfunction in two patients treated with atezolizumab (an antiPD-L1 antibody) and combination therapy of ipilimumab and nivolumab, respectively (Table 1) [26,27]. It is unknown whether APAs are positive at baseline or become positive before the onset of pituitary dysfunction; thus, further studies are needed to clarify the utility of APAs as a biomarker of pituitary irAEs.

Susceptibility alleles of human leucocyte antigen (HLA) have been reported in patients with pituitary dysfunction induced by ICIs. One study that analyzed 11 cases of pituitary dysfunction (both hypophysitis and IAD) induced by ICIs reported that the frequencies of HLA-DR15, -B52, and -Cw12 were higher in patients with pituitary dysfunction than in healthy individuals from Japan [28]. Another study reported that the frequencies of HLA-DQB1*06:01, DPB1*09:01, and -DRB5*01:02 were higher in patients with IAD induced by an anti-PD-1 antibody

Table 1. Autoantibodies Reported in Patients with Endocrine Immune-Related Adverse Events

\begin{tabular}{|c|c|c|c|}
\hline Type of endocrine irAE & Clinical type & At baseline $^{a}$ & At onset ${ }^{b}$ \\
\hline \multirow[t]{2}{*}{ Pituitary dysfunction } & Hypophysitis & Unknown & APA \\
\hline & $\mathrm{IAD}$ & Unknown & APA \\
\hline Primary adrenal insufficiency & & Unknown & 21-Hydroxylase Ab \\
\hline \multirow[t]{3}{*}{ Thyroid dysfunction } & Destructive thyroiditis & TgAb, TPOAb & TgAb, TPOAb \\
\hline & Hypothyroidism & TgAb, TPOAb & $\mathrm{TgAb}, \mathrm{TPOAb}$ \\
\hline & Hyperthyroidism & Unknown & TRAb \\
\hline Hypoparathyroidism & & Unknown & Anti-CaSR Ab \\
\hline Type 1 diabetes mellitus & & GADAb, IA-2Ab, ZnT8Ab & GADAb, IA-2Ab, ZnT8Ab, IAA, ICA \\
\hline
\end{tabular}


$(n=8)$ compared with healthy individuals in Japan [29]. These candidate HLA susceptibility alleles need to be validated in a larger number of cases and in both clinical types of pituitary dysfunction (hypophysitis and IAD).

\section{PRIMARY ADRENAL INSUFFICIENCY}

\section{Epidemiology}

Primary adrenal insufficiency induced by ICIs is a rare adverse event, with an incidence of only $0.7 \%$ (43/5,831 patients), according to a systematic review of 62 study cohorts [11]. That review also reported that the incidence of primary adrenal insufficiency is higher after combination therapy with anti-CTLA-4 and anti-PD-1 antibodies $(4.2 \% ; 11 / 262)$. Primary adrenal insufficiency has been reported after treatment with anti-CTLA-4 [30], anti-PD-1 [31], and anti-PD-L1 [11] antibodies. The period of greatest susceptibility to developing primary adrenal insufficiency remains unknown.

\section{Symptoms}

The following symptoms are observed in patients who develop primary adrenal insufficiency induced by ICIs: general fatigue, tiredness, weakness, weight loss, anorexia, digestive symptoms, loss of muscle strength, impaired consciousness, psychiatric symptoms, and decreased blood pressure [3].

\section{Endocrinological assessments}

Endocrinological tests show decreased levels of serum cortisol, increased levels of plasma ACTH, and increased levels of plasma renin activity (or renin concentration), resulting in the development of hyponatremia, hyperkalemia, or hypoglycemia [3].

\section{Imaging}

In case reports, abdominal computed tomography (CT) showed bilateral enlargement of the adrenal glands [6,30], while positron emission tomography revealed increased uptake of ${ }^{18} \mathrm{~F}$-fluorodeoxyglucose in the adrenal glands [31]. However, primary adrenal insufficiency should be carefully diagnosed because the above radiological findings are also observed in patients with primary or metastatic cancer in the adrenal glands.

\section{Diagnosis}

Primary adrenal insufficiency induced by ICIs is defined as a decreased level of serum cortisol, increased level of plasma $\mathrm{ACTH}$, decreased response of cortisol secretion in the ACTH stimulation test, and a normal ACTH response to corticotropin- releasing hormone [3].

\section{Treatments and outcomes}

If adrenal insufficiency is suspected, hydrocortisone (10 to 20 $\mathrm{mg}$ /day) should be administered immediately for cortisol deficiency [32]. There is no evidence of an effect of high glucocorticoid doses on the recovery of adrenal gland dysfunction or enlargement. The presence of hyponatremia, hypotension, or saltwasting suggests a mineral corticoid deficiency, which can be treated with fludrocortisone in combination with hydrocortisone [3]. Once the general conditions stabilize after the appropriate treatments, ICIs can be considered for patients with primary adrenal insufficiency. There are no reports of an association between the development of primary adrenal insufficiency and ICI treatment outcomes.

\section{Pathology and mechanisms}

Primary adrenal insufficiency induced by ICIs may result from inflammation in the adrenal glands. However, no studies have evaluated the pathological characteristics of the adrenal glands in patients who develop primary adrenal insufficiency induced by ICIs.

\section{Biomarkers}

Although 21-hydroxylase autoantibody positivity was reported in a patient who developed adrenal dysfunction induced by the anti-PD-L1 antibody atezolizumab (Table 1) [27], the utility of the 21-hydroxylase antibody as a biomarker of primary adrenal insufficiency induced by ICIs is unknown.

\section{THYROID DYSFUNCTION}

\section{Epidemiology}

Thyroid dysfunction can be caused by all ICI types, including anti-CTLA-4, anti-PD-1, and PD-L1 antibodies. A systematic review reported that the incidences of hypothyroidism and thyrotoxicosis were $3.8 \%$ and $1.7 \%$ after treatment with an antiCTLA-4 antibody, 7.0\% and 3.2\% after treatment with an antiPD-1 antibody, 3.9\% and $0.6 \%$ after treatment with an anti-PDL1 antibody, and $13.2 \%$ and $8.0 \%$ after combination therapy with anti-CTLA-4 and anti-PD-1 antibodies, respectively [11]. The incidence of thyroid dysfunction was significantly higher after treatment with anti-PD-1 antibodies or anti-CTLA-4/antiPD-1 combination therapy compared with anti-CTLA-4 antibodies [11]. 


\section{Clinical types}

Thyroid dysfunction induced by ICIs can be classified into thyrotoxicosis and hypothyroidism [3]. The main cause of thyrotoxicosis is destructive thyroiditis, which consists of transient thyrotoxicosis followed by hypothyroidism $[33,34]$. In contrast, the development of hyperthyroidism (Graves' disease) after ICI treatment is quite rare [35,36]. Thyroid dysfunction is usually observed 2 to 6 weeks after ICI administration [3]. In our prospective study evaluating the effect of anti-PD-1 antibodies on the development of endocrine irAEs in 209 patients, 12 $(57.4 \%)$, seven $(33.5 \%)$, and one $(0.5 \%)$ patient developed destructive thyroiditis, hypothyroidism without a prior thyrotoxicosis phase, and hyperthyroidism, respectively [35]. Although the severity of thyroid dysfunction, according to the CTCAE 5.0 criteria, is usually low, there was one case of thyroid storm induced by ipilimumab plus nivolumab combination therapy [37].

\section{Symptoms}

The following symptoms are observed in patients with thyrotoxicosis: general fatigue, palpitations, sweating, weight loss, fever, diarrhea, and tremor [3]. In contrast, the symptoms of hypothyroidism include general fatigue, weight gain, constipation, anorexia, and bradycardia [3].

\section{Endocrinological assessments}

Patients who develop destructive thyroiditis induced by ICIs show suppressed levels of serum TSH, elevated levels of serum FT4 and/or free triiodothyronine, and negativity for anti-TSH receptor antibodies [3]. Patients are clinically diagnosed with hyperthyroidism if they show thyrotoxicosis with positivity for anti-TSH receptor antibodies [36] and are definitively diagnosed if they show increased uptake of radionuclides, including ${ }^{99 \mathrm{~m}} \mathrm{Tc}$ pertechnetate, in the thyroid [35]. Patients with hypothyroidism induced by ICIs show increased serum TSH and decreased serum FT4 levels [3].

\section{Imaging}

Thyroid ultrasonography shows hypoechogenicity and/or an irregular echo pattern in patients with destructive thyroiditis and hypothyroidism induced by ICIs. Thyroid scintigraphy shows decreased uptake of radionuclides in destructive thyroiditis and hypothyroidism but increased uptake in hyperthyroidism.

\section{Diagnosis}

Thyrotoxicosis induced by ICIs is defined as a suppressed serum TSH level and elevated serum FT4 and/or free triiodothy- ronine levels. Hypothyroidism induced by ICIs is defined as increased serum TSH and decreased serum FT4 levels.

\section{Treatments and outcomes}

Symptoms caused by thyrotoxicosis are relieved by $\beta$ blockers. Anti-thyroid drugs can be considered in patients who develop hyperthyroidism [3]. Hypothyroidism is treated with levothyroxine, starting at 25 to $50 \mu \mathrm{g} /$ day $(12.5 \mu \mathrm{g} /$ day in the elderly or patients with cardiac diseases). The levothyroxine dose should be adjusted according to the serum TSH level [3]. A retrospective study showed no positive effect of high glucocorticoid doses on thyroid dysfunction [38]. Once the general conditions have stabilized after appropriate treatment, the use of ICIs can be considered for patients with thyroid dysfunction. Some studies have shown an association between thyroid dysfunction development and ICI treatment outcomes [8-10,39].

\section{Pathology and mechanisms}

One report showed, as a histopathological feature, infiltration of cytotoxic $\mathrm{T}$ cells $\left(\mathrm{CD} 8^{+}\right)$in the thyroid gland of a patient who developed thyroid dysfunction induced by the anti-PD-1 antibody nivolumab [40]. Given that the incidence of thyroid dysfunction is higher in patients with anti-thyroid antibody positivity, compared with negativity, at baseline $[33,35,41]$, pre-existing autoimmunity in the thyroid may be involved in the pathogenesis of thyroid dysfunction induced by ICIs. However, the contribution of cellular and/or humoral immunity remains unknown.

\section{Biomarkers}

Anti-thyroid antibodies, including anti-thyroglobulin antibodies and anti-thyroid peroxidase antibodies, are positive in some patients who develop thyroid dysfunction not only at the onset of thyroid dysfunction but also at baseline (before the initiation of ICIs) (Table 1) [9,33-35,41,42]. Our prospective study revealed that the incidence of thyroid dysfunction induced by anti-PD-1 antibodies was higher in patients with anti-thyroid antibody positivity $(34.1 \% ; 15 / 44)$ compared with negativity $(2.4 \%$; 4/165) [35]. Furthermore, among the patients with positive antithyroid antibodies, a much higher incidence of thyroid dysfunction was observed in those with an irregular versus regular echo pattern in the thyroid glands $(56.5 \%$ [13/23] vs. $5.3 \%$ [1/19]) [35], suggesting that the risk of thyroid dysfunction can be predicted by evaluating anti-thyroid antibodies followed by thyroid ultrasonography. In a retrospective study, multivariate logistic regression analysis showed that anti-thyroglobulin antibody 
positivity at baseline and an elevated TSH level $(>5 \mu \mathrm{IU} / \mathrm{mL})$ were independent risk factors for thyroid dysfunction induced by nivolumab [41]. Another retrospective study reported that increased uptake of $18 \mathrm{~F}$-fluorodeoxyglucose in the thyroid glands on positron emission tomography was associated with a higher incidence of thyroid dysfunction induced by nivolumab [10].

\section{HYPOPARATHYROIDISM}

\section{Epidemiology}

Hypoparathyroidism has been reported in patients treated with an anti-PD-1 antibody [43-45] or combination therapy with anti-CTLA-4 and anti-PD-1 antibodies [46-48]. However, the number of case reports is limited and the incidence of hypoparathyroidism unknown.

\section{Symptoms}

Hypocalcemia is associated with neuromuscular symptoms (numbness in the limbs or tetany) and convulsions [3].

\section{Endocrinological assessments}

Endocrinological tests show decreased levels of serum intact parathyroid hormone (PTH), hypocalcemia, and hyperphosphatemia.

\section{Imaging}

There are no reports showing imaging data from patients who developed hypoparathyroidism induced by ICIs.

\section{Diagnosis}

Hypoparathyroidism induced by ICIs is defined as a decreased serum intact PTH level, hypocalcemia, and hyperphosphatemia [3].

\section{Treatments and outcomes}

In patients who require emergency medical care, hypocalcemia should be treated by intravenous administration of calcium gluconate [3]. In patients who do not require emergency medical care, hypocalcemia can be treated by oral administration of active vitamin D [3]. There is no reported evidence for an effect of high-dose glucocorticoids on the recovery of parathyroid function. Once the general conditions are stabilized after appropriate treatments, the use of ICIs can be considered for patients with hypoparathyroidism. There are no studies showing the association of the development of hypoparathyroidism with outcomes of ICI treatment.

\section{Pathology and mechanisms}

The pathological characteristics of the parathyroid glands in patients with ICI-induced hypoparathyroidism have not been reported. Although it is possible that hypoparathyroidism induced by ICIs results from inflammation in the parathyroid glands, several case reports have demonstrated the presence of anti-calcium-sensing receptor antibodies, suggesting the involvement of humoral immunity in the pathogenesis of ICI-induced hypoparathyroidism $[43,45,47]$.

\section{Biomarkers}

Although several case reports detected functional autoantibodies against calcium-sensing receptor (Table 1) [43,45,47], it remains unknown if these antibodies can serve as biomarkers of hypoparathyroidism induced by ICIs.

\section{TYPE 1 DIABETES MELLITUS}

\section{Epidemiology}

Type 1 diabetes mellitus (T1DM) is a rare, yet potentially lifethreatening, endocrine irAE. According to a systematic review, the incidence of T1DM development after ICI treatment is $0.2 \%$ [11]. Although most reported cases of T1DM are associated with anti-PD-1 or anti-PD-L1 antibody treatment $[49,50]$, there is a report of T1DM developing after anti-CTLA-4 antibody (ipilimumab) monotherapy [51].

\section{Clinical types}

ICI treatment can cause acute-onset as well as fulminant T1DM [49]. Fulminant T1DM is a subtype of T1DM first reported in Japan and is characterized by diabetic ketoacidosis and rapid destruction of pancreatic $\beta$ cells [52]. In a case series involving 22 Japanese patients who developed T1DM induced by ICIs, $50 \%$ of the patients fulfilled the criteria of fulminant T1DM [49]. The median duration from the initiation of ICI treatment to the development of T1DM is 5 to 6 months, but the range is 1 week to over 1 year $[49,50]$.

\section{Symptoms}

The symptoms of hyperglycemia include thirst, polydipsia, and polyuria. In addition, ketosis or ketoacidosis causes general fatigue and impaired consciousness or coma [3].

\section{Endocrinological assessments}

Glucose levels are increased in the blood and urine. The hemoglobin A1c level is also elevated, but the increase may be rela- 
tively small in cases of rapid development of T1DM. Ketone bodies may be elevated in the blood and urine. C-peptide levels are decreased or gradually decreased in serum and urine even when endogenous insulin secretion is partially preserved at onset [49].

\section{Imaging}

There are no reports of imaging data from patients who developed T1DM induced by ICIs.

\section{Diagnosis}

The diagnostic criteria for acute-onset and fulminant T1DM have been established by the Committee of the Japan Diabetes Society $[53,54]$. Patients who develop ketosis or diabetic ketoacidosis within 3 months after the onset of hyperglycemic symptoms and who require insulin treatment continuously after diabetes diagnosis are diagnosed with acute-onset T1DM. Fulminant T1DM is diagnosed when the following three criteria are fulfilled: (1) diabetic ketosis or ketoacidosis developing soon (approximately 7 days) after the onset of hyperglycemic symptoms; (2) plasma glucose level $\geq 16.0 \mathrm{mmol} / \mathrm{L}$ ( $\geq 288 \mathrm{mg} / \mathrm{dL})$ and hemoglobin A1c level $<8.7 \%$ (National Glycohemoglobin Standardization Program value) at first visit; and (3) urinary Cpeptide excretion $<10 \mu \mathrm{g} /$ day or fasting serum C-peptide level $<0.3 \mathrm{ng} / \mathrm{mL}$ and serum C-peptide level after intravenous glucagon (or after meal) load $<0.5 \mathrm{ng} / \mathrm{mL}$ at onset [54].

\section{Treatments and outcomes}

Patients who develop T1DM induced by ICIs require insulin treatment. Patients showing ketosis or ketoacidosis should be treated with intravenous administration of insulin and saline. If ketosis is not observed at onset or not improved after treatment, patients can be managed by intensive insulin therapy (subcutaneous injections) [3]. Since glucocorticoids can exacerbate blood glucose control, and there is no evidence of the effect of glucocorticoids on the recovery of insulin secretion, glucocorticoids are not recommended for the treatment of T1DM [3]. Once the general conditions stabilize after the appropriate treatments, the use of ICIs can be considered for patients with T1DM. No studies have evaluated the association between T1DM development and ICI treatment outcomes.

\section{Pathology and mechanisms}

A case report described the pathological features of the pancreas in a patient who developed T1DM after ipilimumab and nivolumab combination therapy followed by nivolumab [55].
Histopathological analysis of this patient showed infiltration of $\mathrm{CD}^{+} \mathrm{T}$ cells not only around the pancreatic islets but also throughout the pancreas. Among the T cell populations in the pancreas, the number of $\mathrm{CD}^{+} \mathrm{T}$ cells was dominant over that of $\mathrm{CD}^{+} \mathrm{T}$ cells [55]. These findings suggest the involvement of cytotoxic T cells in the development of ICI-induced T1DM.

\section{Biomarkers}

In a case series analyzing autoantibodies associated with T1DM in 27 patients who developed ICI-induced T1DM (Caucasians, $n=24$; non-Hispanics, $n=1$; Asians, $n=1$; non-Hispanic and other races, $n=1)$, the prevalences of anti-glutamic acid decarboxylase (GAD), anti-islet antigen 2 (IA2), anti-zinc transporter 8 , and islet cell antibodies were $36 \%$ (9/25 patients), $21 \%$ (5/24), 10\% (2/20), and 11\% (2/19), respectively (Table 1) [50]. In contrast, a study of Japanese patients who developed T1DM induced by ICIs reported that only one of $20(5 \%)$ patients was positive for anti-GAD antibodies [49], suggesting that the prevalence of autoantibodies associated with T1DM varies among races. Interestingly, positivity for anti-GAD, anti-IA2, and antizinc transporter 8 antibodies before the initiation of ICI treatment was reported in a patient with ICI-induced T1DM (Table 1) [50], suggesting that these autoantibodies at baseline may be a biomarker of T1DM development in a subset of patients. In addition, a case series reported that the prevalence of HLA-DR4 was higher in patients with ICI-induced T1DM $(76 \%, 16 / 21)$ than in U.S. Caucasians (17.3\%) [50]. Although HLA susceptibility alleles may be useful biomarkers, further studies involving more cases are needed to clarify this.

\section{CONCLUSIONS}

Endocrine irAEs are one of the most frequent adverse events induced by ICIs. Since most symptoms are not specific, oncologists must understand the clinical characteristics of each endocrine irAE to manage it appropriately. The development of endocrine irAEs may result in life-threatening consequences, including adrenal crisis and diabetic ketoacidosis, but it is sometimes associated with better ICI treatment outcomes, especially in patients with pituitary or thyroid dysfunction. Therefore, management of endocrine irAEs contributes not only to treatment of endocrine dysfunction but also to better ICI treatment outcomes. Identification of specific biomarkers of individual endocrine irAEs will improve the outcomes of cancer immunotherapy using ICIs. 


\section{CONFLICTS OF INTEREST}

Shintaro Iwama is a consultant/advisory board member on endocrinological adverse events for Ono Pharmaceutical Company, Bristol-Myers Squibb, and Chugai Pharmaceutical Co. Ltd., and received personal fees from Ono Pharmaceutical Company, Bristol-Myers Squibb, Chugai Pharmaceutical Co., Ltd., and MSD K.K outside of this study. Hiroshi Arima received grants from Ono Pharmaceutical Company, MSD K.K., and Chugai Pharmaceutical Co. Ltd., as well as personal fees from Ono Pharmaceutical Company, Bristol-Myers Squibb, and MSD K.K outside of this study.

\section{ORCID}

Shintaro Iwama https://orcid.org/0000-0002-3281-0337

Hiroshi Arima https://orcid.org/0000-0003-3746-1997

\section{REFERENCES}

1. Brahmer JR, Lacchetti C, Schneider BJ, Atkins MB, Brassil KJ, Caterino JM, et al. Management of immune-related adverse events in patients treated with immune checkpoint inhibitor therapy: American Society of Clinical Oncology clinical practice guideline. J Clin Oncol 2018;36:1714-68.

2. Puzanov I, Diab A, Abdallah K, Bingham CO 3rd, Brogdon C, Dadu R, et al. Managing toxicities associated with immune checkpoint inhibitors: consensus recommendations from the Society for Immunotherapy of Cancer (SITC) Toxicity Management Working Group. J Immunother Cancer 2017;5:95.

3. Arima H, Iwama S, Inaba H, Ariyasu H, Makita N, Otsuki $\mathrm{M}$, et al. Management of immune-related adverse events in endocrine organs induced by immune checkpoint inhibitors: clinical guidelines of the Japan Endocrine Society. Endocr J 2019;66:581-6.

4. Zhai Y, Ye X, Hu F, Xu J, Guo X, Zhuang Y, et al. Endocrine toxicity of immune checkpoint inhibitors: a real-world study leveraging US Food and Drug Administration adverse events reporting system. J Immunother Cancer 2019;7:286.

5. Byun DJ, Wolchok JD, Rosenberg LM, Girotra M. Cancer immunotherapy: immune checkpoint blockade and associated endocrinopathies. Nat Rev Endocrinol 2017;13:195-207.

6. Chang LS, Barroso-Sousa R, Tolaney SM, Hodi FS, Kaiser UB, Min L. Endocrine toxicity of cancer immunotherapy targeting immune checkpoints. Endocr Rev 2019;40:17-65.
7. Faje AT, Lawrence D, Flaherty K, Freedman C, Fadden R, Rubin K, et al. High-dose glucocorticoids for the treatment of ipilimumab-induced hypophysitis is associated with reduced survival in patients with melanoma. Cancer 2018;124: 3706-14.

8. Kobayashi T, Iwama S, Yasuda Y, Okada N, Okuji T, Ito M, et al. Pituitary dysfunction induced by immune checkpoint inhibitors is associated with better overall survival in both malignant melanoma and non-small cell lung carcinoma: a prospective study. J Immunother Cancer 2020;8:e000779.

9. Osorio JC, Ni A, Chaft JE, Pollina R, Kasler MK, Stephens $\mathrm{D}$, et al. Antibody-mediated thyroid dysfunction during $\mathrm{T}$ cell checkpoint blockade in patients with non-small-cell lung cancer. Ann Oncol 2017;28:583-9.

10. Yamauchi I, Yasoda A, Matsumoto S, Sakamori Y, Kim YH, Nomura M, et al. Incidence, features, and prognosis of immune-related adverse events involving the thyroid gland induced by nivolumab. PLoS One 2019;14:e0216954.

11. Barroso-Sousa R, Barry WT, Garrido-Castro AC, Hodi FS, Min L, Krop IE, et al. Incidence of endocrine dysfunction following the use of different immune checkpoint inhibitor regimens: a systematic review and meta-analysis. JAMA Oncol 2018;4:173-82.

12. Caturegli P, Di Dalmazi G, Lombardi M, Grosso F, Larman HB, Larman T, et al. Hypophysitis secondary to cytotoxic Tlymphocyte-associated protein 4 blockade: insights into pathogenesis from an autopsy series. Am J Pathol 2016;186: 3225-35.

13. Dillard T, Yedinak CG, Alumkal J, Fleseriu M. Anti-CTLA-4 antibody therapy associated autoimmune hypophysitis: serious immune related adverse events across a spectrum of cancer subtypes. Pituitary 2010;13:29-38.

14. Zhao C, Tella SH, Del Rivero J, Kommalapati A, Ebenuwa I, Gulley J, et al. Anti-PD-L1 treatment induced central diabetes insipidus. J Clin Endocrinol Metab 2018;103:365-9.

15. Okano Y, Satoh T, Horiguchi K, Toyoda M, Osaki A, Matsumoto $\mathrm{S}$, et al. Nivolumab-induced hypophysitis in a patient with advanced malignant melanoma. Endocr J 2016;63: 905-12.

16. Cho KY, Miyoshi H, Nakamura A, Kurita T, Atsumi T. Hyponatremia can be a powerful predictor of the development of isolated ACTH deficiency associated with nivolumab treatment [Letter to the Editor]. Endocr J 2017;64:235-6.

17. Kanie K, Iguchi G, Bando H, Fujita Y, Odake Y, Yoshida K, et al. Two cases of atezolizumab-induced hypophysitis. J Endocr Soc 2017;2:91-5. 
18. Min L, Hodi FS, Giobbie-Hurder A, Ott PA, Luke JJ, Donahue $\mathrm{H}$, et al. Systemic high-dose corticosteroid treatment does not improve the outcome of ipilimumab-related hypophysitis: a retrospective cohort study. Clin Cancer Res 2015;21:749-55.

19. Iwama S, De Remigis A, Callahan MK, Slovin SF, Wolchok JD, Caturegli P. Pituitary expression of CTLA-4 mediates hypophysitis secondary to administration of CTLA-4 blocking antibody. Sci Transl Med 2014;6:230ra45.

20. Iwama S, Arima H. Anti-pituitary antibodies as a marker of autoimmunity in pituitary glands. Endocr J 2020;67:107783.

21. Tahir SA, Gao J, Miura Y, Blando J, Tidwell RSS, Zhao H, et al. Autoimmune antibodies correlate with immune checkpoint therapy-induced toxicities. Proc Natl Acad Sci U S A 2019;116:22246-51.

22. Ricciuti A, De Remigis A, Landek-Salgado MA, De Vincentiis L, Guaraldi F, Lupi I, et al. Detection of pituitary antibodies by immunofluorescence: approach and results in patients with pituitary diseases. J Clin Endocrinol Metab 2014; 99:1758-66.

23. Iwata N, Iwama S, Sugimura Y, Yasuda Y, Nakashima K, Takeuchi S, et al. Anti-pituitary antibodies against corticotrophs in IgG4-related hypophysitis. Pituitary 2017;20:30110 .

24. Lupi I, Manetti L, Raffaelli V, Grasso L, Sardella C, Cosottini M, et al. Pituitary autoimmunity is associated with hypopituitarism in patients with primary empty sella. J Endocrinol Invest 2011;34:e240-4.

25. Iwama S, Welt CK, Romero CJ, Radovick S, Caturegli P. Isolated prolactin deficiency associated with serum autoantibodies against prolactin-secreting cells. J Clin Endocrinol Metab 2013;98:3920-5.

26. Lupi I, Brancatella A, Cosottini M, Viola N, Lanzolla G, Sgro D, et al. Clinical heterogeneity of hypophysitis secondary to PD-1/PD-L1 blockade: insights from four cases. Endocrinol Diabetes Metab Case Rep 2019;2019:EDM-190102.

27. Lanzolla G, Coppelli A, Cosottini M, Del Prato S, Marcocci C, Lupi I. Immune checkpoint blockade anti-PD-L1 as a trigger for autoimmune polyendocrine syndrome. J Endocr Soc 2019;3:496-503.

28. Yano S, Ashida K, Sakamoto R, Sakaguchi C, Ogata M, Maruyama K, et al. Human leucocyte antigen DR15, a possible predictive marker for immune checkpoint inhibitor-induced secondary adrenal insufficiency. Eur J Cancer 2020;
130:198-203.

29. Inaba H, Ariyasu H, Iwakura H, Ueda Y, Kurimoto C, Uraki $\mathrm{S}$, et al. Comparative analysis of human leucocyte antigen between idiopathic and anti-PD-1 antibody induced isolated adrenocorticotropic hormone deficiency: a pilot study. Clin Endocrinol (Oxf) 2019;91:786-92.

30. Min L, Ibrahim N. Ipilimumab-induced autoimmune adrenalitis. Lancet Diabetes Endocrinol 2013;1:e15.

31. Trainer H, Hulse P, Higham CE, Trainer P, Lorigan P. Hyponatraemia secondary to nivolumab-induced primary adrenal failure. Endocrinol Diabetes Metab Case Rep 2016;2016:EDM16-0108.

32. Yanase T, Tajima T, Katabami T, Iwasaki Y, Tanahashi Y, Sugawara A, et al. Diagnosis and treatment of adrenal insufficiency including adrenal crisis: a Japan Endocrine Society clinical practice guideline [Opinion]. Endocr J 2016;63:765-84.

33. Kobayashi T, Iwama S, Yasuda Y, Okada N, Tsunekawa T, Onoue T, et al. Patients with antithyroid antibodies are prone to develop destructive thyroiditis by nivolumab: a prospective study. J Endocr Soc 2018;2:241-51.

34. Yamauchi I, Sakane Y, Fukuda Y, Fujii T, Taura D, Hirata M, et al. Clinical features of nivolumab-induced thyroiditis: a case series study. Thyroid 2017;27:894-901.

35. Okada N, Iwama S, Okuji T, Kobayashi T, Yasuda Y, Wada E, et al. Anti-thyroid antibodies and thyroid echo pattern at baseline as risk factors for thyroid dysfunction induced by anti-programmed cell death-1 antibodies: a prospective study. Br J Cancer 2020;122:771-7.

36. Kurihara S, Oikawa Y, Nakajima R, Satomura A, Tanaka R, Kagamu H, et al. Simultaneous development of Graves' disease and type 1 diabetes during anti-programmed cell death-1 therapy: a case report. J Diabetes Investig 2020;11:1006-9.

37. McMillen B, Dhillon MS, Yong-Yow S. A rare case of thyroid storm. BMJ Case Rep 2016;2016:214603.

38. Ma C, Hodi FS, Giobbie-Hurder A, Wang X, Zhou J, Zhang A, et al. The impact of high-dose glucocorticoids on the outcome of immune-checkpoint inhibitor-related thyroid disorders. Cancer Immunol Res 2019;7:1214-20.

39. Basak EA, van der Meer JWM, Hurkmans DP, Schreurs MWJ, Oomen-de Hoop E, van der Veldt AAM, et al. Overt thyroid dysfunction and anti-thyroid antibodies predict response to anti-PD-1 immunotherapy in cancer patients. Thyroid 2020;30:966-73.

40. Neppl C, Kaderli RM, Trepp R, Schmitt AM, Berger MD, Wehrli M, et al. Histology of nivolumab-induced thyroiditis. Thyroid 2018;28:1727-8. 
41. Kimbara S, Fujiwara Y, Iwama S, Ohashi K, Kuchiba A, Arima $\mathrm{H}$, et al. Association of antithyroglobulin antibodies with the development of thyroid dysfunction induced by nivolumab. Cancer Sci 2018;109:3583-90.

42. Kotwal A, Haddox C, Block M, Kudva YC. Immune checkpoint inhibitors: an emerging cause of insulin-dependent diabetes. BMJ Open Diabetes Res Care 2019;7:e000591.

43. Piranavan P, Li Y, Brown E, Kemp EH, Trivedi N. Immune checkpoint inhibitor-induced hypoparathyroidism associated with calcium-sensing receptor-activating autoantibodies. J Clin Endocrinol Metab 2019;104:550-6.

44. Umeguchi H, Takenoshita H, Inoue H, Kurihara Y, Sakaguchi C, Yano S, et al. Autoimmune-related primary hypoparathyroidism possibly induced by the administration of pembrolizumab: a case report. J Oncol Pract 2018;14:449-51.

45. Lupi I, Brancatella A, Cetani F, Latrofa F, Kemp EH, Marcocci C. Activating antibodies to the calcium-sensing receptor in immunotherapy-induced hypoparathyroidism. J Clin Endocrinol Metab 2020;105:dgaa092.

46. Win MA, Thein KZ, Qdaisat A, Yeung SJ. Acute symptomatic hypocalcemia from immune checkpoint therapy-induced hypoparathyroidism. Am J Emerg Med 2017;35:1039.

47. Dadu R, Rodgers TE, Trinh VA, Kemp EH, Cubb TD, Patel $\mathrm{S}$, et al. Calcium-sensing receptor autoantibody-mediated hypoparathyroidism associated with immune checkpoint inhibitor therapy: diagnosis and long-term follow-up. J Immunother Cancer 2020;8:e00687.

48. Trinh B, Sanchez GO, Herzig P, Laubli H. Inflammation-induced hypoparathyroidism triggered by combination immune checkpoint blockade for melanoma. J Immunother Cancer 2019;7:52.
49. Baden MY, Imagawa A, Abiru N, Awata T, Ikegami H, Uchigata $Y$, et al. Characteristics and clinical course of type 1 diabetes mellitus related to anti-programmed cell death-1 therapy. Diabetol Int 2018;10:58-66.

50. Stamatouli AM, Quandt Z, Perdigoto AL, Clark PL, Kluger $\mathrm{H}$, Weiss SA, et al. Collateral damage: insulin-dependent diabetes induced with checkpoint inhibitors. Diabetes 2018; 67:1471-80.

51. Tsiogka A, Jansky GL, Bauer JW, Koelblinger P. Fulminant type 1 diabetes after adjuvant ipilimumab therapy in cutaneous melanoma. Melanoma Res 2017;27:524-5.

52. Imagawa A, Hanafusa T, Miyagawa J, Matsuzawa Y. A novel subtype of type 1 diabetes mellitus characterized by a rapid onset and an absence of diabetes-related antibodies. Osaka IDDM Study Group. N Engl J Med 2000;342:301-7.

53. Kawasaki E, Maruyama T, Imagawa A, Awata T, Ikegami H, Uchigata $Y$, et al. Diagnostic criteria for acute-onset type 1 diabetes mellitus (2012): report of the committee of Japan Diabetes Society on the research of fulminant and acute-onset type 1 diabetes mellitus. J Diabetes Investig 2014;5:115-8.

54. Imagawa A, Hanafusa T, Awata T, Ikegami H, Uchigata Y, Osawa H, et al. Report of the committee of the Japan Diabetes Society on the research of fulminant and acute-onset type 1 diabetes mellitus: new diagnostic criteria of fulminant type 1 diabetes mellitus (2012). J Diabetes Investig 2012;3:536-9.

55. Yoneda S, Imagawa A, Hosokawa Y, Baden MY, Kimura T, Uno S, et al. T-lymphocyte infiltration to islets in the pancreas of a patient who developed type 1 diabetes after administration of immune checkpoint inhibitors. Diabetes Care 2019; 42:e116-8. 